\title{
Dielectric behaviour of strontium tartrate single crystals
}

\author{
S K ARORA*, VIPUL PATEL, BRIJESH AMIN and ANJANA KOTHARI \\ Department of Physics, Sardar Patel University, Vallabh Vidyanagar 388 120, India
}

MS received 10 February 2003; revised 9 January 2004

\begin{abstract}
Strontium tartrate trihydrate (STT) crystals have been grown in silica hydrogel. Various polarization mechanisms such as atomic polarization of lattice, orientational polarization of dipoles and space charge polarization in the grown crystals have been understood using results of the measurements of dielectric constant $\left(\varepsilon^{\prime}\right)$ and dielectric loss $(\tan \delta)$ as functions of frequency and temperature. Ion core type polarization is seen in the temperature range $75-180^{\circ} \mathrm{C}$, and above $180^{\circ} \mathrm{C}$, there is interfacial polarization for relatively lower frequency range. One observes dielectric dispersion at lower frequency presumably due to domain wall relaxation.
\end{abstract}

Keywords. STT crystals; dielectric polarization; tangent loss; dehydration.

\section{Introduction}

In recent years, owing to a number of practical applications in the field of micro-electronics and opto-electronics, a great deal of interest has been shown in the study of the dielectric behaviour of various materials (Gould and Bowler 1988; Ismail and Gould 1989; Jin et al 1993; Raghavendra et al 2002). Strontium tartrate is an important ferroelectric material with device applications (Rahimkutty et al 2001). Nonlinear optical devices based on second harmonic generation, crystal oscillators and rescuators, and devices to central laser emission are some of these applications. It has also been identified as a strategic material with a global business potential (Selvasekarapandian et al 1999). TGA and DTA studies on STT (Rahimkutty et al 2001) have concluded that water molecules are locked up with different strengths in the crystal lattice. Vibrational studies, based on IR and Raman spectra, of the gel-grown STT have revealed the molecular structure of tartrate, monohydrotartrate and water indents.

Since the data on dielectric properties of strontium tartrate trihydrate (STT) do not exist in the literature, we present in this paper our investigations based on measurements of the dependence of dielectric constant as well as tangent loss on applied frequency and environmental temperature as external parameter.

\section{Experimental}

Strontium tartrate trihydrate $\left(\mathrm{SrC}_{4} \mathrm{H}_{4} \mathrm{O}_{6} \cdot 3 \mathrm{H}_{2} \mathrm{O}\right)$ samples in the form of single crystals were prepared by employing

*Author for correspondence controlled ionic diffusion through acidified silica hydrogel (Henisch 1970; Arora 1981). We obtained pale yellowish crystal touching the bottom of tubes, along with transparent and faceted crystal within the gel column. The yellowish crystals were strontium tartrate tetrahydrate, but our study here refers to the well-faceted, transparent strontium tartrate trihydrate (STT) crystals of size between $3 \times 2 \times 0.5 \mathrm{~mm}^{3}$ and $20 \times 12 \times 5 \mathrm{~mm}^{3}$. They are nonhygroscopic structures, and predominantly exhibit the three basic morphological forms of pinacoids and prisms with the dominant (100), (010) and (001) faces. Crystallinity of the grown crystal was confirmed by X-ray diffraction. The crystal is found to be a monoclinic perovskite structure with space group $P 2_{1}$. The computed unit cell dimensions are

$$
\begin{array}{lll}
a=0.755 \mathrm{~nm}, & b=1.006 \mathrm{~nm}, & c=0.647 \mathrm{~nm} \\
\alpha=90^{\circ}, & \beta=102^{\circ}, & \gamma=90^{\circ}
\end{array}
$$

Their EDAX trace showed prominent strontium peak, and their pycnometric density was found to be $2 \cdot 054 \mathrm{~g} \cdot \mathrm{cm}^{-3}$. For the present study, a crystal with prominent (100) grown face was mounted between a pair of flat stainless steel electrodes. The capacitance was measured in the temperature range $27-421^{\circ} \mathrm{C}$ using a specially constructed sample holder shown in figure 1. It may be noted that the holder was not evacuated. The variation in temperature was carried out after enclosing the cell in a resistance heated muffle furnace, while the sample temperature was monitored using a chromel-alumel thermocouple $\left( \pm 1^{\circ} \mathrm{C}\right)$. The temperature was increased by regulating the input power through 'AE' make 15-amp dimmerstat, the rate of heating was maintained linear $\left(40^{\circ} \mathrm{C} / \mathrm{h}\right)$. The capacitance measurements were made using 'Hewlett Packard' 4284A LCR meter. The measured values of the capacitance were converted into dielectric constant and dissipation factor by determining the area and thickness of the crystal. 


\section{Results and discussion}

The data on measurements in respect of temperature and frequency dependence of both dielectric constant and the dissipation factor of STT single crystals have been plotted graphically in figures $2-5$. The temperature dependence of dielectric constant and $\tan \delta$ in the present case manifests pyroelectric behaviour of the crystals. Some interesting observations revealed from figures 2-5 are noted as follows.

The values of both $\varepsilon^{\prime}$ and $\tan \delta$ are sensitive to temperature as well as frequency. One can easily notice two peaks on the smooth background, one at $42^{\circ} \mathrm{C}$ and the other at $179^{\circ} \mathrm{C}$. In the low temperature range of $42-75^{\circ} \mathrm{C}, \varepsilon^{\prime}$ and $\tan \delta$ markedly decrease, whereas between $75^{\circ}$ and $179^{\circ} \mathrm{C}$, these are essentially temperature independent. In the higher temperature region, i.e. beyond $180^{\circ} \mathrm{C}$, they become strongly temperature dependent, increasing faster with increasing temperature and with decreasing applied frequency. Evidently, the material exhibits dielectric dispersion in this region. Furthermore, beyond a frequency of $50 \mathrm{kHz}, \varepsilon^{\prime}$ and $\tan \delta$ become nearly frequency independent. In the whole temperature region studied, i.e. $29-421^{\circ} \mathrm{C}$, both these parameters continuously gradually decrease with increase in the applied frequency. In the range $29-180^{\circ} \mathrm{C}$, the decrement in $\varepsilon^{\prime}$ and tan $\delta$ is faster at lower temperatures, whereas above $180^{\circ} \mathrm{C}$ the decrement is faster at higher temperatures. Evidently, the dielectric dispersion in the material is again supported. It may be mentioned that the measurements of $\varepsilon^{\prime}$ and/or tan $\delta$ by reversing the temperature cycle could not be carried out because the crystal begins to undergo thermal phase transition at $75^{\circ} \mathrm{C}$.

The dielectric constant vs temperature plot (figure 2) shows a small peak at $42^{\circ} \mathrm{C}$, indicating suspected ferroelectric transition. A plot of $\varepsilon^{\prime}$ vs $1 /\left(T-T_{\mathrm{c}}\right)$ with frequency as a parameter is presented in figure 6 , where $T_{\mathrm{c}}$ is the critical temperature $\left(42^{\circ} \mathrm{C}\right)$. Below the transition temperature, $T_{\mathrm{c}}$, the dielectric constant, $\varepsilon^{\prime}$, of the compound follows Curie-Weiss law

$$
\varepsilon^{\prime}=\frac{A}{T-T_{\mathrm{c}}}, \tan \delta=\frac{\omega\left(\alpha+\beta T+\gamma T^{2}\right)}{T-T_{\mathrm{c}}},
$$

where $A$ is the Curie constant, $\alpha$ the harmonic and defect contribution, $\beta$ and $\gamma$ arise due to anharmonic interaction of the lattice. The material loses intrinsic polarization above $T_{\mathrm{c}}$ and becomes paraelectric (Matthew 2000). An illustrative expression for the total dielectric constant, for instance, at $500 \mathrm{~Hz}$ frequency, chosen from the curve in figure 6 , is written as

$$
\varepsilon^{\prime}=7 \cdot 78+\frac{12 \cdot 64}{T-42} \text {. }
$$

Evidently, the dielectric constant (figure 2) has contributions (Rupprecht and Bell 1964) from two effects,

$$
\varepsilon^{\prime}=\varepsilon_{\mathrm{P}}^{\prime}+\varepsilon_{\mathrm{CW}}^{\prime} .
$$

The first term, viz. $\varepsilon_{\mathrm{P}}^{\prime}$, is temperature independent and it arises from the polarization of the ions themselves, whereas the second term, viz. $\varepsilon_{\mathrm{CW}}^{\prime}$, is temperature dependent
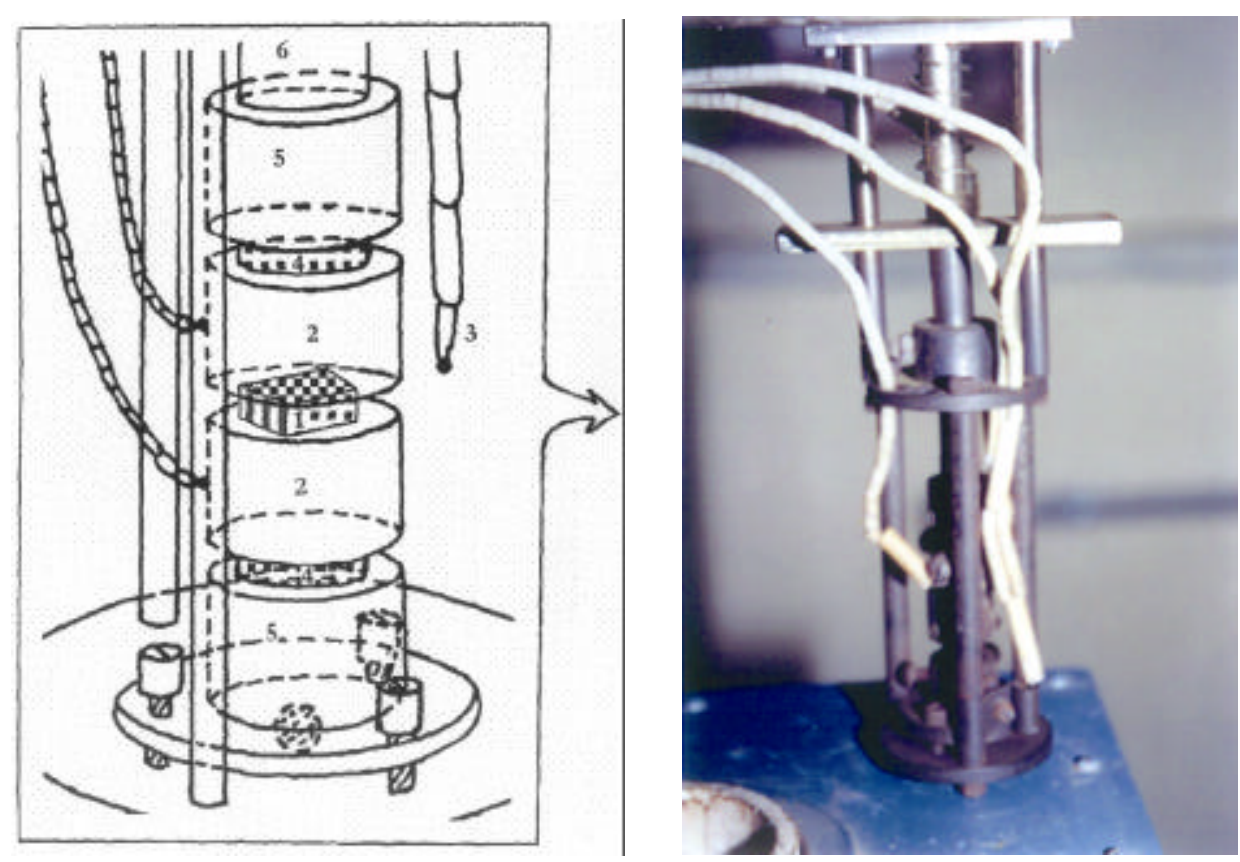

Figure 1. Design of sample holder used for measurements. Evacuation of the holder is not required. 1. Sample, 2. electrodes, 3. thermocouple, 4. porcelain plate, 5. connector and 6. metal rod. 
and it is the contribution from the movement of ions from their equilibrium position.

The dielectric constant of the dispersive medium after $42^{\circ} \mathrm{C}$ is observed to be decreasing. This might be because the term contributing to dielectric constant resulting from the ion-dipole interactions is compensated by thermal energy, leading to the relaxation of polarization. In the temperature range $75-180^{\circ} \mathrm{C}, \varepsilon^{\prime}$ remains almost temperatureindependent, which indicates ion core type of polarization in the material. Beyond $180^{\circ} \mathrm{C}$, the dielectric capacitance (and hence $\varepsilon^{\prime}$ ) is found to increase with tem- perature, faster with lower frequency (refer curves with frequency, $\leq 5 \mathrm{kHz}$ ). This may be ascribed to interfacial polarization mechanism. The charge carriers as existing can migrate some distance under the influence of applied fields. When such carriers are blocked at the cathode, space charge region develops which might be responsible for substantial increase in $\varepsilon^{\prime}$ toward lower frequencies.

When the lattice thermal energy is of the order of iondipole interaction, the contacts between water molecules and the effective ionic charges will break. Therefore, one would expect dielectric anomaly to exist. Indeed one can

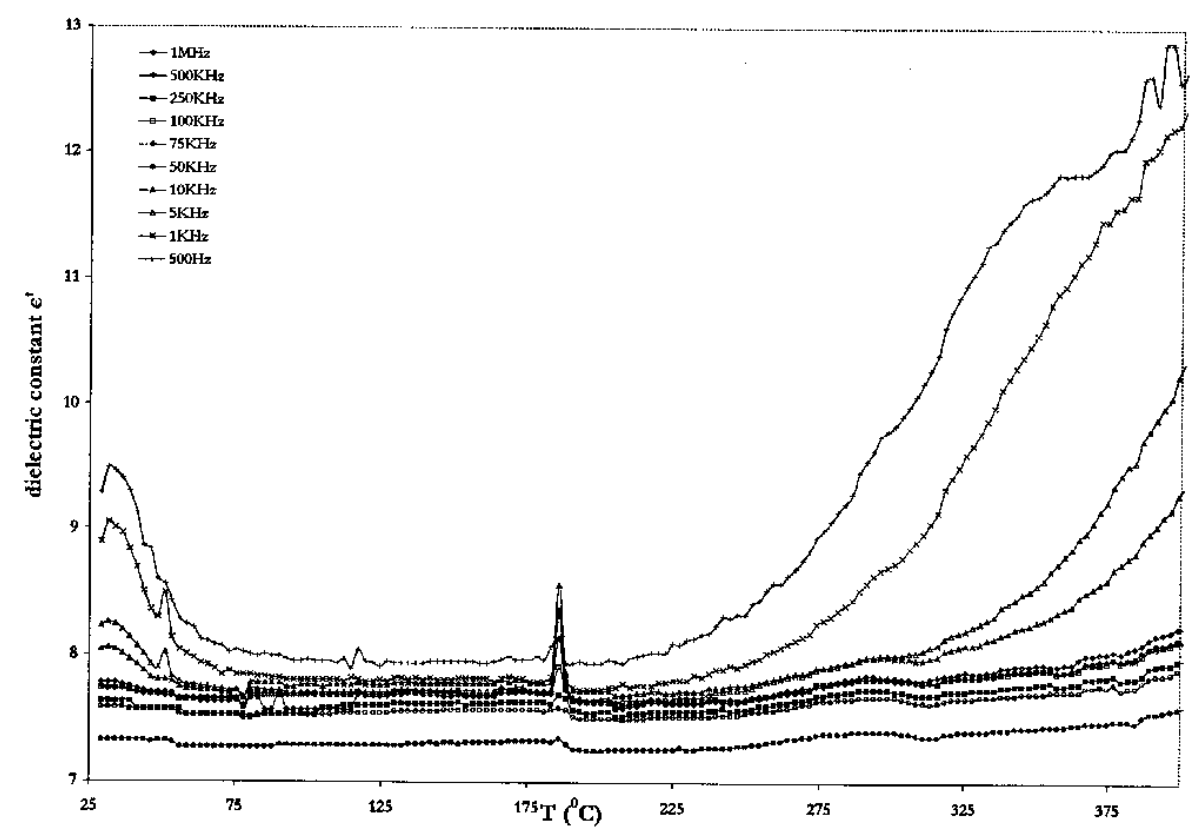

Figure 2. Graphical presentation of the variation of dielectric constant with temperature.

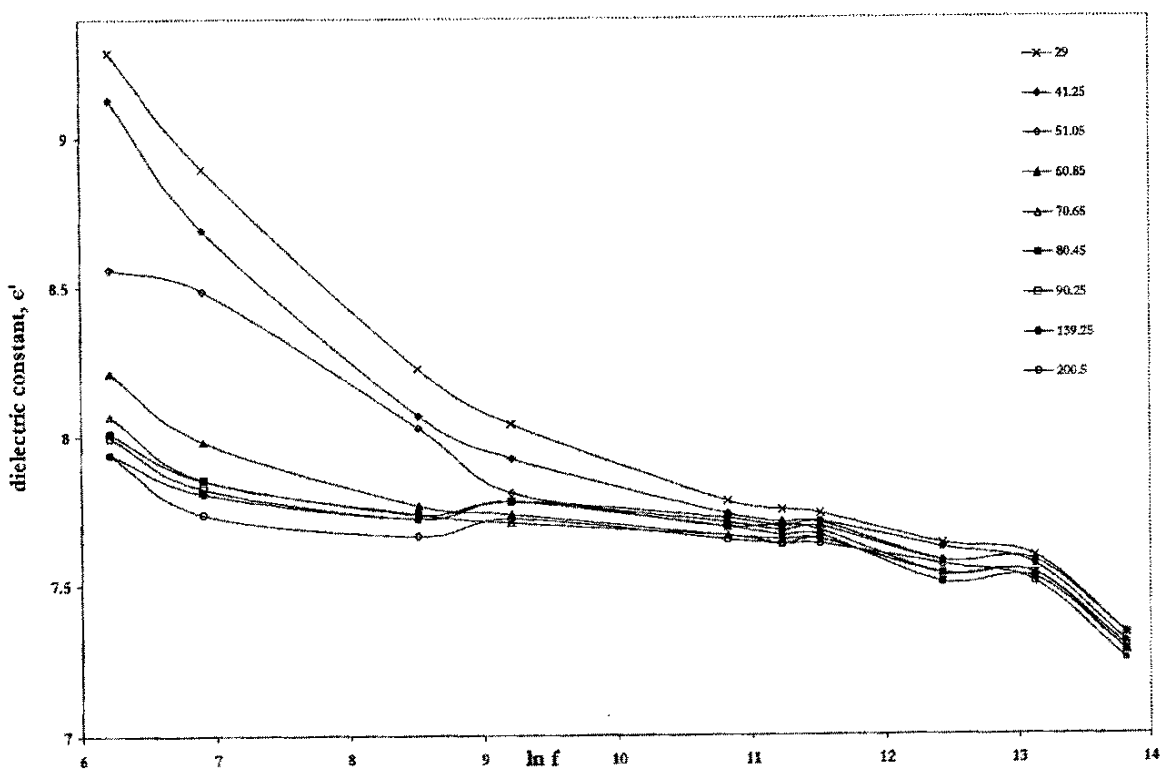

Figure 3. Frequency dependence of dielectric constant. 
see a sharp, quite noticeable peak at $179^{\circ} \mathrm{C}$, which may be attributed to liberation of three water molecules of crystallization from the lattice. This process of dehydration has been confirmed from TGA and DTA analyses (Arora et al 2004). As the temperature of STT increases, the applied thermal energy is enough to produce dehydration of the crystals, allowing thermally generated charge carriers to develop enough space charge polarization. This explains the observed relatively faster increase of $\varepsilon^{\prime}$ as well as $\tan \delta$ in the high temperature region. At such higher temperatures the polarization increases because of the ease of accumulation of excess charge carriers in the absence

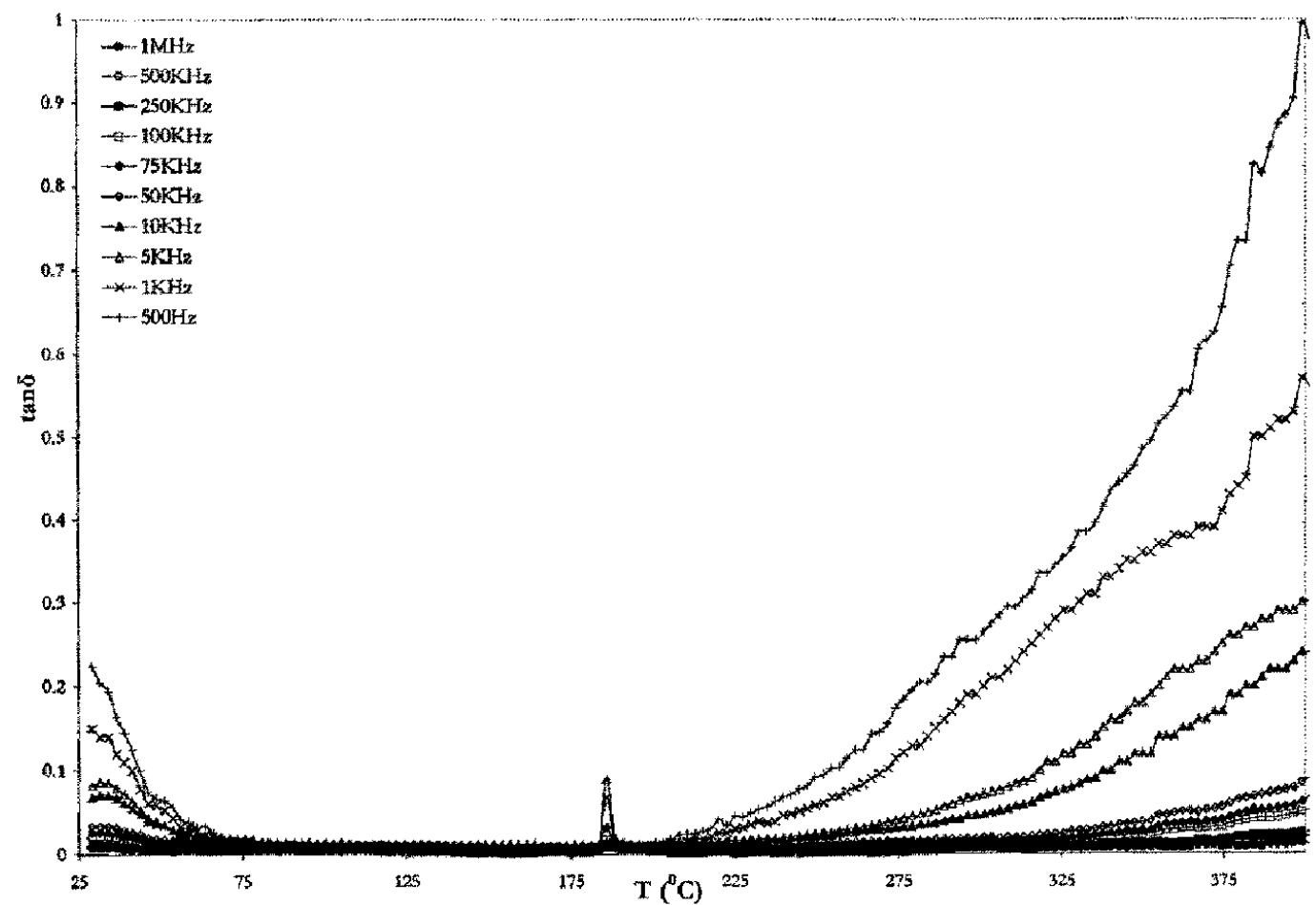

Figure 4. Graphical presentation of the variation of $\tan \delta$ with temperature.

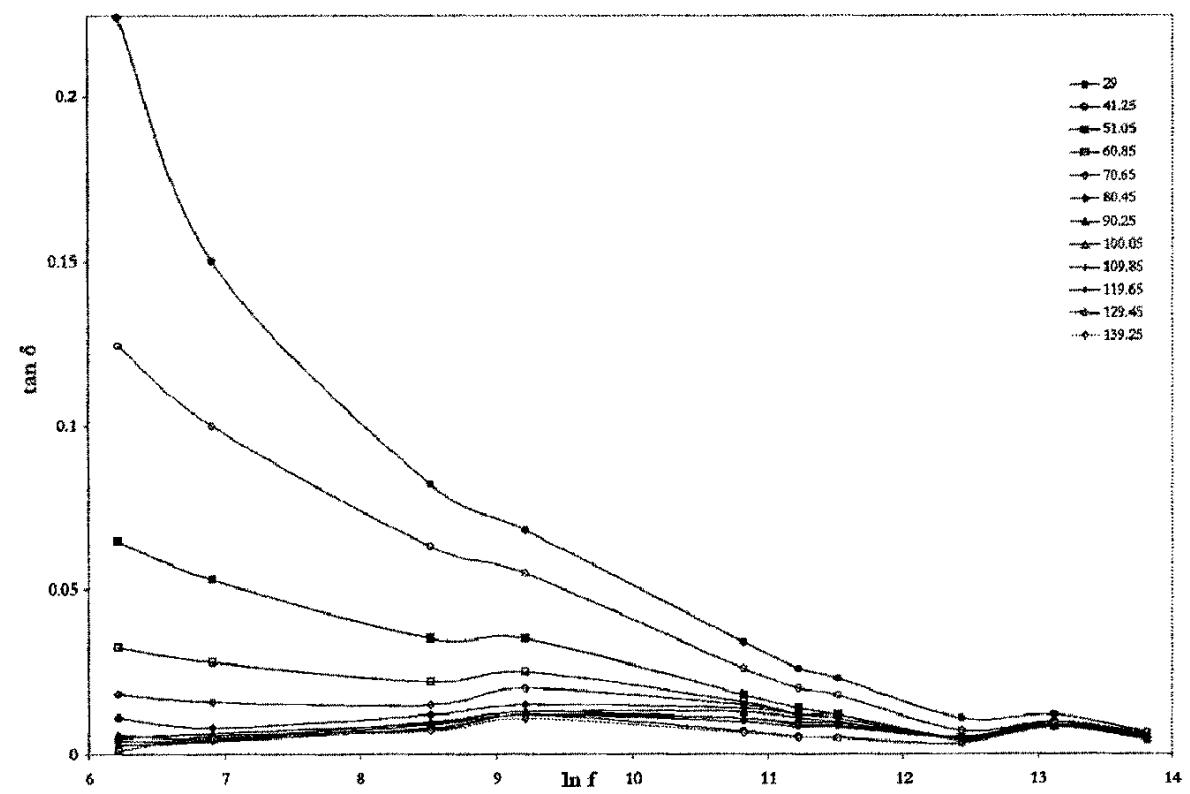

Figure 5. Frequency dependence of $\tan \delta$. 
of ion-dipole contacts. An ionic crystal with higher dielectric constant shows lower energy of formation of lattice defects (Samara 1984). The defects (e.g. vacancies) can cause positive or negative space charge distribution which can move under application of an external field and when they are trapped by the defects, larger number of dipoles are formed, giving rise to space charge polarization.

Phonon generation at high temperature is expected to raise $\varepsilon^{\prime}$ due to phonon drag. Phonon drag does not localize charge carriers, exhibiting atomic polarizability and physical expansion of lattice. Consequently, in the temperature range $180-250^{\circ} \mathrm{C}$ there occurs relatively slower increase of $\varepsilon^{\prime}$, as observed. The rapid increase of $\varepsilon^{\prime}$ and $\tan \delta$ beyond $250^{\circ} \mathrm{C}$ may be because of conducting nature of the compound (large d.c. conductivity), on account of availability of $\mathrm{H}^{+}$and $\mathrm{OH}^{-}$ions.

Now, we look at the variation of $\varepsilon^{\prime}$ and $\tan \delta$ with frequency. Broadly speaking, figure 3 manifests that the dielectric constant and tangent losses are both inversely proportional to frequency. This is a normal dielectric behaviour (Anderson 1964), that both $\varepsilon^{\prime}$ and $\tan \delta$ (figures 2 and 4) decrease with increasing frequency. This can be understood on the basis that the mechanism of polarization is similar to that of conduction process. The electronic exchange of the number of ions in the crystals gives local displacement of electrons in the direction of applied field that gives polarization. As the frequency increases, a point will be reached where the space charge cannot sustain and comply the external field and hence the polarization decreases, giving rise to diminishing values of $\varepsilon^{\prime}$ (figure 3). Evidently, dielectric dispersion is noticeable at lower frequency and lower temperature.
At relatively lower frequency, the higher the temperature (figure 3), the larger is the dielectric constant. Such variations at higher temperature may be attributed to the blocking of charge carriers at the electrodes. Because of impedance to their motion at the electrodes, space charge and macroscopic distortion results, which might cause the observed larger values of $\varepsilon^{\prime}$ at lower frequencies. Further, the obvious and quite distinct anomaly near the transition temperature can be explained by the fact that the optical mode phonon frequency softens near the transition temperature, thus giving much larger values of $\varepsilon^{\prime}$ and $\tan \delta$, as manifested by noticeable peaks.

With increasing temperature (figure 3 ), a high degree of dispersion in the permittivity begins to occur at lower frequency $(\leq 50 \mathrm{kHz})$. This could be again due to the activated (thermally generated) charge carriers from the onset of space charge limited d.c. conduction (Mansingh 1980). Succinctly, the space charge effect does lead to the dispersion of $\varepsilon^{\prime}$ at low frequencies, and particularly at higher temperatures, because of the enhanced mobility of charge carriers (Hippel 1954; Kingery et al 1976). In our crystals, $\varepsilon^{\prime}$ decreases rapidly with increasing frequency and tends to reach a constant value (at around $10 \mathrm{kHz}$ ) depending on the fact that beyond this frequency of electric field the electron exchange does not follow the alternating field. Continuous gradual decrease in $\tan \delta$ as well as $\varepsilon^{\prime}$ with frequency suggests that STT, like any normal dielectric, may have domains of different sizes and varying relaxation times. One can speculate three possible reasons for this dispersion: (a) dipole orientations within the domain as can be found in many order-disorder ferroelectrics (Sandy and Jones 1968), (b) Maxwell-Wagner dis-

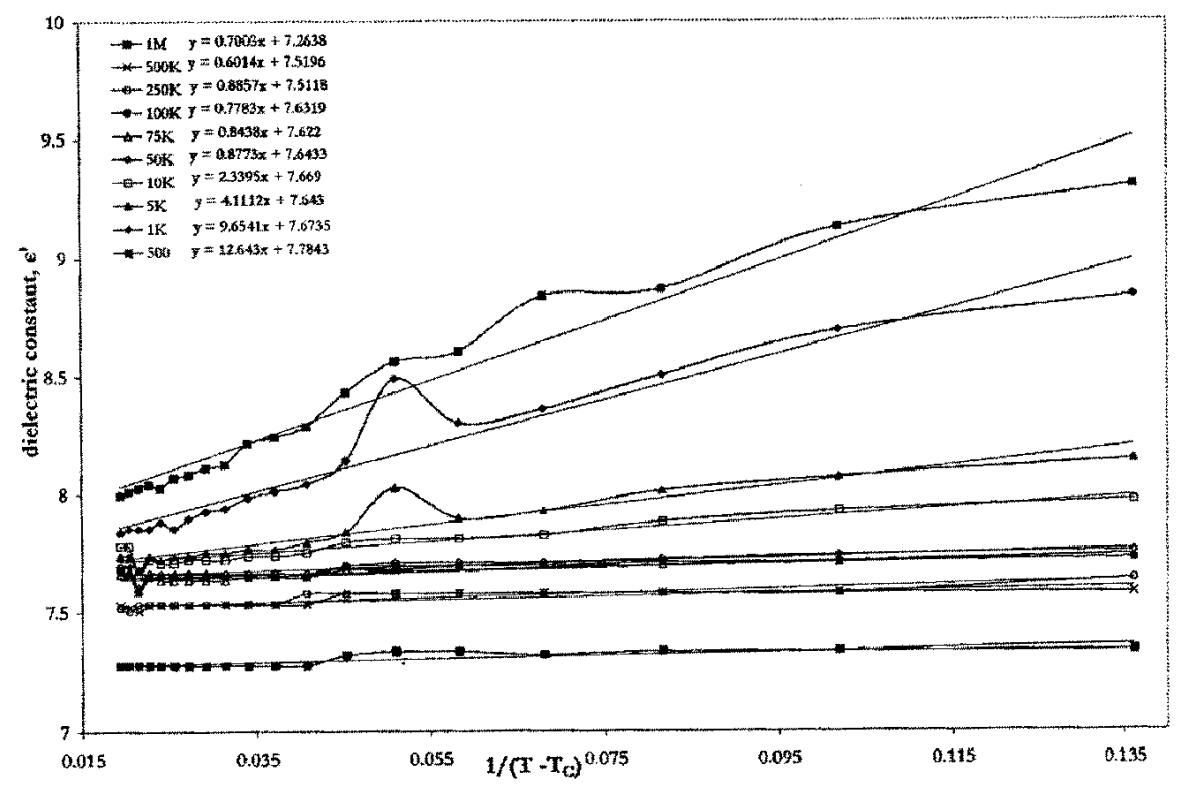

Figure 6. Dielectric constant, $\varepsilon^{\prime}$ vs $1 /\left(T-T_{\mathrm{c}}\right)$. 


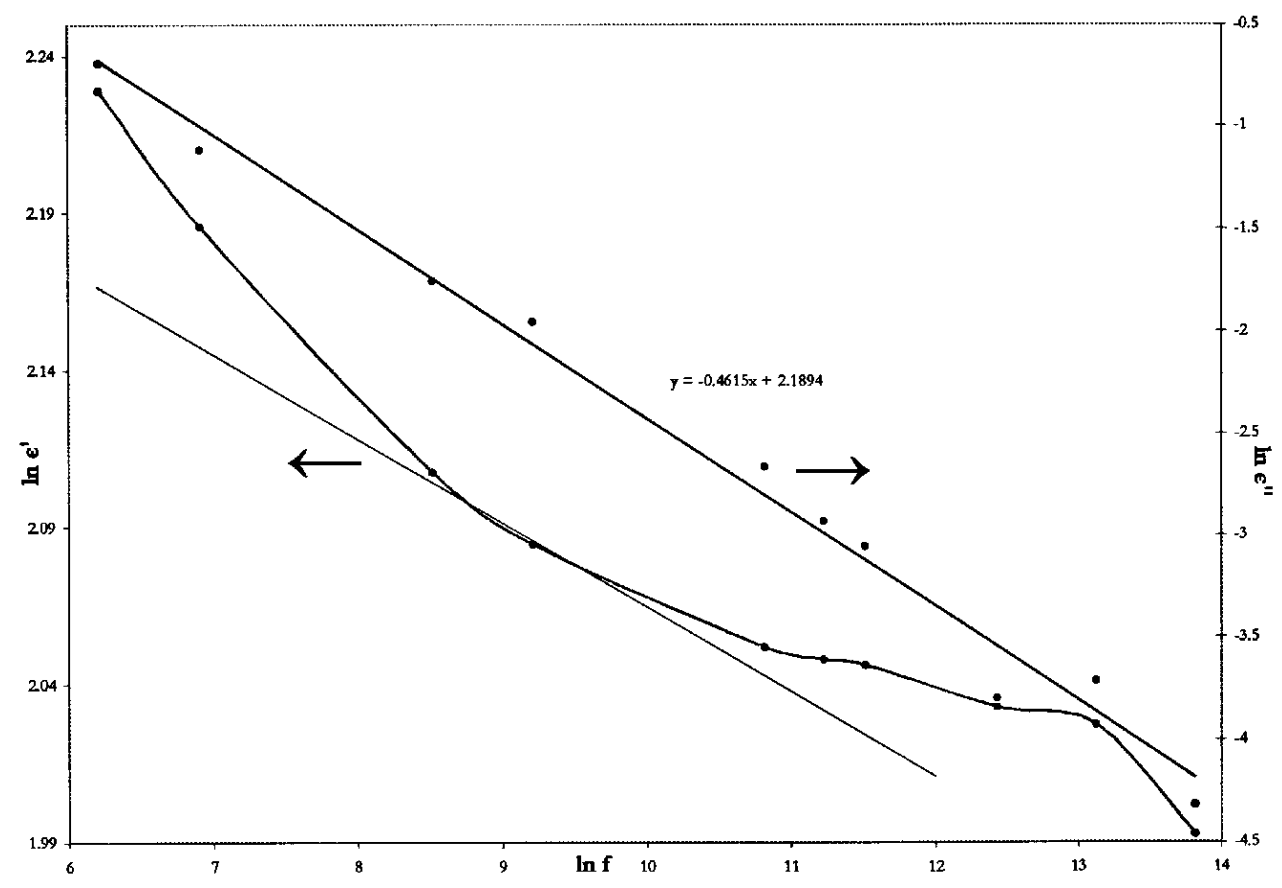

Figure 7. Graphical plot of $\ln \varepsilon^{\prime}$ and $\ln \varepsilon^{\prime \prime}$ vs $\ln \mathrm{f}$.

persion due to surface effects (Schlosser and Drougard 1961; Mansingh et al 1977), and (c) dipolar relaxation. For Maxwell-Wagner dispersion a relaxation frequency of $1 \mathrm{kHz}$ requires, with a maximum of $100 \mathrm{pF}$ bulk capacitance, a bulk resistance of the order $\leq 10^{5} \Omega$ which is much lesser than the observed resistance $\left(10^{9} \Omega\right.$, as measured using 'MEGGER' megohmeter) of STT crystals. So the possibility of low frequency dispersion via Maxwell-Wagner effect is to be ruled out, considering the resistivity range. For dipolar relaxation, one would expect slowing down behaviour and hence increased dielectric relaxation times. However, from figures 3 and 5, it is evident that the relaxation frequency increases or the relaxation time decreases, approaching the transition temperature of $42^{\circ} \mathrm{C}$. This rules out the dipolar relaxation also. With both dipolar and Maxwell-Wagner relaxations ruled out, the low frequency dispersion is probably caused by domain wall relaxation. The relaxation time decreases with increasing temperature for domain wall relaxation (Gurk 1972). The exact mechanism of domain wall relaxation cannot be established here. Nevertheless, the arguments given above are strongly indicative of this particular mechanism to be applicable in the present case.

The dissipation factor, $D=\tan \delta=\varepsilon^{\prime \prime} / \varepsilon^{\prime}$, is an important parameter of the material which is convenient to keep as lower as possible for practical reasons (Cereceda et al 1998). The imaginary and real parts of the permittivity at room temperature as function of frequency are presented on a double logarithmic scale as shown in figure 7 . The straight lines represent the theoretical fit, based on the validity of the universal dynamics law (Jonscher 1977), expressed by

$$
\varepsilon^{\prime \prime}(\omega)=\cot (n \pi / 2) \cdot \varepsilon^{\prime}(\omega)=K \omega^{n-1}
$$

where $K$ is a positive constant of proportionality.

Figure 7 illustrates that the frequency exponent, $n$, is equal to $0 \cdot 524$. That this value is very small physically reflects the level of nonideality for the capacitance involved. The deviation of material capacitance from an ideal behaviour is a clear evidence of the existence of intrinsic defects. Since $\varepsilon^{\prime \prime}(\omega)$ is interpreted as the contribution of conductivity meaning the lost energy, a high loss can be expected for a small $n$ value. This approach is in excellent agreement with figure 7 as well as the high dissipation factor (Lanfredi et al 2000).

\section{Conclusions}

Dielectric constant, $\varepsilon^{\prime}$, and dissipation factor, $\tan \delta$, of strontium tartrate trihydrate single crystals show similar trend of variation with frequency as well as temperature. A ferroelectric transition occurs at $42^{\circ} \mathrm{C}$, while crystal dehydration occurs at $179^{\circ} \mathrm{C}$. As the temperature goes much beyond the transition temperature, dielectric dispersion becomes more evident $(<10 \mathrm{kHz})$. Beyond $250^{\circ} \mathrm{C}$, sharp increase of dielectric constant is due to space charge polarization, compatible with higher d.c. conductivity. Continuous fall of the dielectric constant with increasing frequency occurs because dipoles are not able to follow faithfully the impressed oscillating field. Interestingly, our 
crystal is not an ideal capacitor, its ideality factor being as small as 0.524 .

\section{References}

Anderson J C 1964 Dielectrics (London: Chapman and Hall) p. 16

Arora S K 1981 Prog. Cryst. Growth Charact. 4345

Arora S K, Patel Vipul and Kothari Anjana 2004 Mater. Chem. Phys. 84323

Cereceda Noe, Noheda Beatriz and Gouzalo J A 1998 Ferroelectric Lett. 23135

Gould R D and Bowler C J 1988 Thin Solid Films 164281

Gurk P 1972 Phys. Status Solidi (a)10 407

Henisch H K 1970 Crystal growth in gels (Pennsylvania, USA: The Pennsylvania State University Press)

Hippel A R von 1954 Dielectrics and waves (New York: Wiley) Ch. II

Ismail B B and Gould R D 1989 Phys. Status Solidi (a)115 237

Jin Byung-Moon, Bhalla A S, Choi Byung-Chun and Kim Jung-Nam 1993 Phys. Status Solidi (a)140 239
Jonscher A K 1977 Nature 267673

Kingery W D, Bowen H K and Uhlmann D R 1976 Introduction to ceramics (New York: Wiley)

Lanfredi S, Carvalho J F and Hernandes A C $2000 \mathrm{~J}$. Appl. Phys. 88283

Mansingh A 1980 Bull. Mater. Sci. 2325

Mansingh A, Srivastava K N and Singh B 1977 J. Phys. D10 1227

Matthew Trainer 2000 Eur. J. Phys. 21459

Raghavendra S C, Raibagkar R L and Kulkarni A B 2002 Bull. Mater. Sci. 2537

Rahimkutty M H, Rajendra Babu K, Shreedharan Pillai K, Sudarsana Kumar M R and Nair C M K 2001 Bull. Mater. Sci. 24249

Rupprecht G and Bell R O 1964 Phys. Rev. 135 A74

Samara G A 1984 Solid State Phys. 181

Sandy F and Jones R V 1968 Phys. Rev. 168481

Schlosser H and Drougard M E 1961 J. Appl. Phys. 32 1227

Selvasekarapandian S, Vivekanandan K and Kolandaivel P 1999 Cryst. Res. Technol. 34873 